\title{
Medicine in China
}

\section{Best of the Old and the New}

\author{
A. J. SMITH
}

British Medical fournal, 1974, 2, 367-370

Last month a party of British doctors went to the People's Republic of China as guests of the Chinese Medical Association, visiting hospitals and medical research institutes in Peking, Shanghai, and Kwanghow. The visit was arranged by the British Council and the Great Britain-China Committee. One of the party was Dr. A. F. Smith, one of the editorial staff of the British Medical Journal. This is the first of a series of articles giving impressions of China; they are the reactions of one individual and do not in any way represent the views of the delegation as a whole.

Perhaps the biggest surprise for the medical visitor to China is the extent of the practical impact of the political philosophy of Chairman Mao Tsetung. At every level the organization of the medical services and the approach in diagnosis and treatment shows obvious evidence of the two major policy directives made at the time of cultural revolution in the late 1960s-that medicine must be taken to the masses and that the best of the old should be combined with the best of the new.

At the hospitals and health stations visited by the British party we were told of the efforts being made to combine Chinese traditional medicine with Western "modern" medicine; this seems to be by far the highest political priority in medicine at the present time.

\section{Historical Background}

When the Communists took over the government of China one of the many problems they faced was the poor state of the health services, disorganized by years of war and based as they were on two conflicting schools of medicine: traditional medicine, with

British Medical Journal, London, WC1H 9JR

A. J. SMITH, B.M., Assistant Editor about 500,000 doctors mostly in rural areas, and Western medicine, with at most 40,000 doctors concentrated in the big cities. ${ }^{1}$

The achievements of the first 15 years of communist rule came under critical examination in the cultural revolution and medicine was the target of two main attacks. The rapid growth of hospitals and medical schools in the cities had, it was said, led to concentration of high quality care in a few centres, and the rural areas had been neglected; and the intellectual elitism of practitioners of Western medicine had prevented the union that should have taken place between the traditional and Western systems.

The first of these criticisms led to a revision of the organization of medical care and increasing emphasis on the role of barefoot doctors*; the second led to powerful political pressures to increase the use of traditional medicine in hospitals and research institutes throughout the country. This process was still under way in the cities we visited on the tour.

\section{Traditional Chinese Medicine}

Chinese traditional medicine can claim to have been the world's first organized body of medical knowledge. ${ }^{2}$ The earliest Chinese medical work, the Huang Ti Nei Ching Su Wen or the Yellow Emperor's Classic of Internal Medicine is said to date back to Huang Ti, emperor between 2698 and 2598 B.C. Certainly the book was written several centuries before the birth of Christ.

The theoretical background of traditional medicine is unique, being concerned with the achievement of balance between the two opposing life forces, yin and yang. Illness is thought to be due to imbalance in these natural forces. Practitioners claim that they can identify specific skin areas which become hypersensitive when the function of an organ is impairedand that the points concerned with a particular organ can be linked to form a line or meridian. The network of these lines and points forms the theoretical basis of the technique of acupuncture.

*'To be considered in a later article. 
Illness should be diagnosable by search for the points which have become hypersensitive; but in practice the process of diagnosis has come to include detailed questioning and observation of the patient and prolonged palpation of the pulse-the main diagnostic procedure of the traditional physician. Traditional physicians that we saw taking medical histories from their patients always seemed to do so with a hand on the pulse. The teaching is that the pulse consists of 6 pulses on each wrist, each connected with a particular organ of the body and each capable of recording very slight abnormalities of the internal organs. By means of the pulse (taken first on the right in women and on the left in men) the physician is said to be able to judge the site and the state of the disease, its cause and duration, whether it is chronic or acute, and whether it will result in death or recovery.

Treatment includes the use of herbal medicines, techniques such as moxibustion (applying heat to the skin by burning a powder, moxa, made from the dried leaves of the plant Artemisia vulgaris) and cupping, exercises, and acupuncture.

\section{Traditional Medicine Today}

Traditional doctors are most numerous-as might be expectedat the level of primary care. In one commune hospital near to the centre of Shanghai the population of 62,000 (factory workers and their families) had 26 doctors, of whom 5 had been trained by traditional methods. In rural areas the ratio was nearer to $50: 50$, while in larger hospitals there seemed to be relatively few traditional practitioners.

The British party visited one medical college, in Kwangchow (Canton), training traditional practitioners. Built in the mid 1950s, its output had been stepped up since the cultural revolution but it still produced only 175 doctors a year while the other medical schools in Kwangchow, training Western-style doctors, have an annual output ten times that number. Though no national statistics were quoted it seems that the proportion of students being trained by traditional methods is small and probably less than $10 \%$. Some formal training in the "opposite" school of medicine is given to doctors, but in practice acquisition of this knowledge seems to be mainly by reading and observation.

The use being made of traditional methods by doctors in China seems to be largely pragmatic. There is no possible reconciliation between the theories of function and disease underlying the two systems-but acting on the principle enunciated by Mao Tsetung that "the past must serve the present" forms of treatment that have proved effective in practice have been adopted by both types of practitioner without any implied acceptance of the theoretical background. The Westerntrained cardiologist uses acupuncture to relieve cardiac pain; the traditional practitioner takes the blood pressure with a standard sphygomanometer though the concept of hypertension is not included in his theories of disease.

\section{Herbal Remedies}

The raw materials of traditional herbal remedies include not only more than 1,000 plants-leaves, fruits, seeds, and nutsbut also such exotic objects as snakes, newts, stalactites, agate, and buffalo horn. The remedies are either given as herbal teas or as mixtures compounded from the raw materials after processing by much the same techniques as those used by herbalists in the west-drying and grounding, boiling, infusing, and shredding. Since the remedies are so widely used, however, there is mass production of the more popular preparations so that while a hospital would have some herbs in their natural state-and every hospital seems to have a herb garden (fig. 1)dispensaries also carry rows of bottles containing mass produced pills and mixtures. Bulk preparation also allows distribution throughout the country of remedies based on plants found in only specific areas.

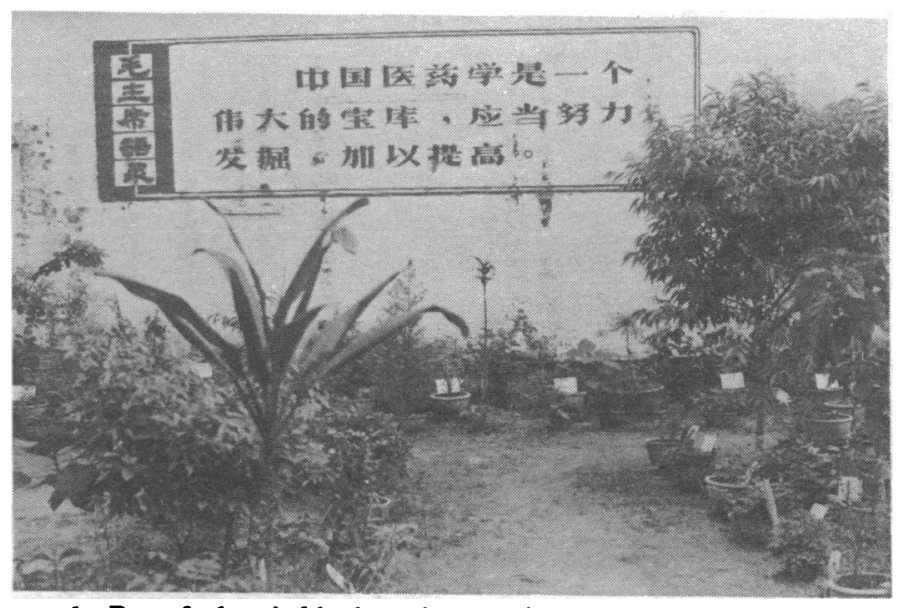

FIG. 1-Part of a hospital herb garden used in the preparation of traditional medicines.

At the level of primary care there is an enormous corpus of empirical knowledge linking remedies with symptoms-azalea flowers for asthma, and so on. People can and do go to the urban chemist's shop and buy over the counter a traditional herbal remedy without consulting a doctor, though there was no suggestion that self-treatment is any more prevalent than it is in Britain. Most herbal remedies are prescribed by traditional doctors, but since the cultural revolution there has been a big increase in their use by Western-trained doctors too. Efforts have also been made in research institutes to establish the content of these remedies and to assess their therapeutic value. The Institute of Materia Medica of the Chinese Academy of Medical Sciences is reputed to be making its way through the "treasurehouse" of traditional medicine, isolating and identifying the active principles.

Clinical trials are under way in many centres. At the Capital Hospital in Peking (fig. 2) we were shown preliminary results of a trial of the management of acute myocardial infarction in 85 patients. The recipe used was one of about 30 said to "improve or accelerate the stagnant blood" and consisted of a peony root and two other plant extracts. All the patients were given conventional Western treatment; those who agreed to take traditional medicine in addition were given the plant extract twice daily. With this method of selection the groups were not strictly comparable but the results were striking: early mortality in those given the traditional medicine was $15 \%$ compared with $31 \%$ in the others, and there was suggestive evidence that the plant

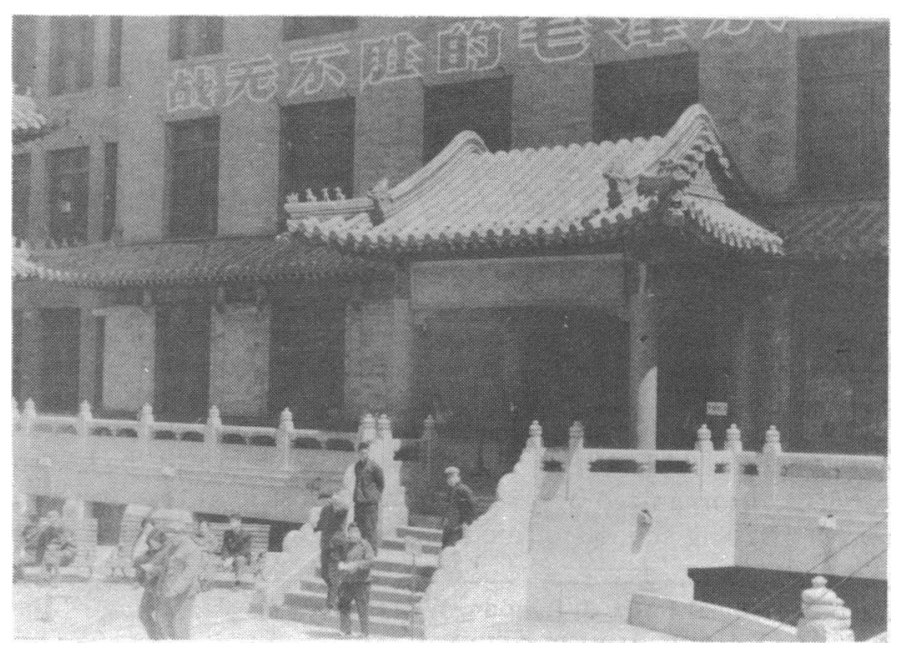

FIG. 2-Main entrance to the Shoutu (Capital) Hospital in Peking. 
medicine had a protective effect against arrythmias and cardiogenic shock. The only side effect reported was some dryness of the mouth.

We were told that this mixture has been evaluated in laboratory tests including the electrocardiographic response to exercise and that it seems to improve performance. Elsewhere on the tour we found other hospitals evaluating traditional medicines in a very wide range of conditions; they are being used for treatment of enuresis, epilepsy, some forms of cancer, and most common heart, lung, and digestive complaints. However, Chinese physicians we met seemed unanimous in their opinion that the use of placebos was unethical-it conflicted with the primary purpose of medicine, "to serve the people," so that comparative trials on a blind basis seem unlikely to be organized in China.

On the other hand traditional medicines are by no means administered uncritically. We were told of many common conditions for which Western medicine is clearly still the first-line treatment. These included fertility control, the treatment of choriocarcinoma (very common in China), and the treatment of schistosomiasis-all conditions for which the essentially symptomatic remedies of traditional medicine would be unlikely to be effective.

\section{Treatment of Fractures without Plaster}

Traditional methods of treating fractures-very different from those in the West - seem to be used in most hospitals in parallel with orthodox Western treatment. In the smaller commune hospitals (fig. 3) we visited the responsibility for treating trauma had been given to one of the traditional doctors, and again this seems common practice. The classical traditional method of treating fractures differs in two ways from that used in Britain. Reduction of the fracture should be achieved slowly, say traditional doctors, and they do this slowly over a period of days, using short wooden splints fixed with bandages over soft paper padding. The splints maintain alignment of the fracture but they are not designed to immobilize the joints above and below it. Indeed the rationale of the treatment is that the muscles and joints around the fracture should retain their mobility; exercise is encouraged very early and the splints are adjusted as often as necessary to cope with the progressive reduction in the swelling around the injury.

In practice only part of the traditional method was followed in the orthopaedic department we visited in the Friendship Hospital in Peking. Firstly, reduction is nowadays achieved in one stage- usually under regional nerve block and radiographic control. Secondly, traditional treatment seems to be given only

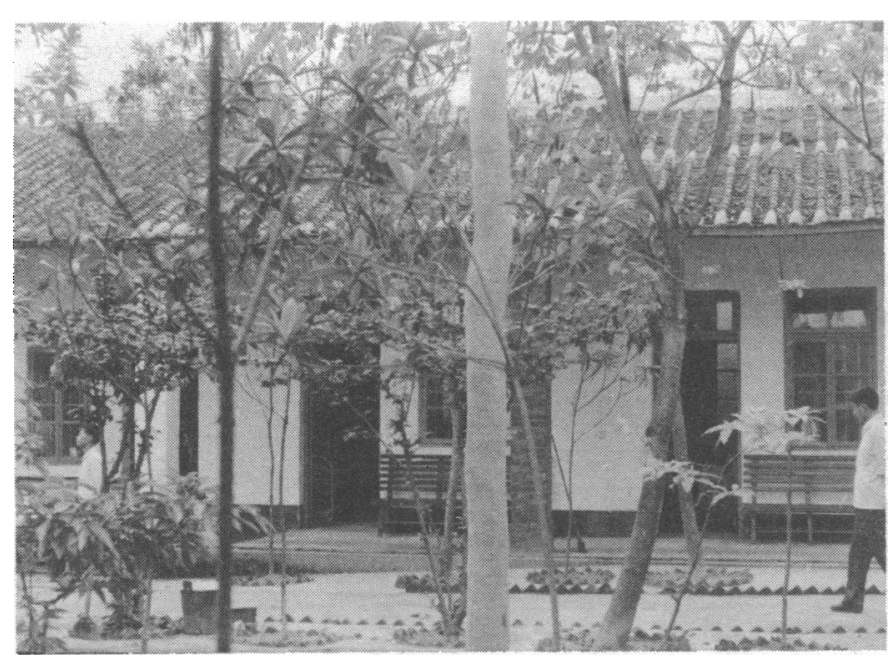

FIG. 3-Inpatient wing of commune hospital outside Kwangchow. for uncomplicated fractures with a good prognosis: examples shown to us were Colles fracture at the wrist and fracture of the shaft of the femur. If the fracture is comminuted, or if it is known to carry a risk of delayed union, plaster is used. One trauma specialist in a rural commune was traditionally trained, and he told us that he sent all fractures of the scaphoid to the district hospital-presumably for immobilization in plaster. What was impressive was the sight of young men with fractures of the femur, treated simply by $4 \mathrm{~kg}$ traction applied via a pin in the tibia without any splint, sitting up in bed and moving freely only four days after the injury.

Several cases we saw had multiple fractures, some of which were immobilized in plaster and others in splints. We were told that in the simple fractures for which it is best used traditional treatment achieves rapid relief of pain and swelling and allows early mobilization; the fractures heal more quickly than those treated in plaster and the functional results are better. Clearly this is yet another branch of medicine in which selection of one or other style of treatment is based on a practical assessment of results rather than on doctrinaire grounds.

\section{Cupping and Moxibustion}

Two of the less well-known techniques of traditional medicine we saw in use are cupping and moxibustion-both examples of the age-old medical treatment of counter-irritation. Cupping is used in China for conditions such as backache, and the procedure is identical with that formerly used in Britain: the mouth of a metal cup is heated and it is then applied to the selected area of the skin. Cooling of the air inside the cup forms a partial vacuum so causing suction and the formation of a haematoma.

Moxibustion seems to be unique to Chinese traditional medicine. At the Traditional Medical College in Kwangchow we saw a woman with a frozen shoulder receiving this treatment. Three acupuncture needles were inserted-one at the tip of the shoulder, one at the elbow, and one at the wrist. A ball of moxa was then applied to the outer end of each needle and ignited. These moxa balls burnt for a few minutes emitting a fair amount of both heat and smoke. Later we saw another patient with sciatica treated by application of local heat from slow burning moxa cartridges. These burn for about 10 minutes and are held an inch or so above the skin. Good results were claimed for each of these procedures: certainly they would all be expected to have an impressive effect on the patient and there is worldwide support for use of both local heat and counter-irritation in musculoskeletal disorders.

\section{Acupuncture}

In the classical system of traditional medicine acupuncture is used in both diagnosis and treatment. Current interest in the West is concentrated on its use in anaesthesia-but in fact this is a very recent newcomer to the scene, first attempted in 1958.

The theoretical basis is the system of acupuncture points distributed along the meridians and each linked with one or more internal organs. At the Traditional Medical College in Kwangchow we were told that there are 361 acupuncture points, but other authorities quote twice as many. The fundamental concept of acupuncture is that detailed knowledge of the distribution of these points allows both diagnosis and treatment: malfunction of an organ may be recognized by hypersensitivity of the corresponding acupuncture points and relieved by stimulation at the irritable point.

\section{Acupuncture in Treatment}

The traditional doctor uses acupuncture to treat many organic disorders and claims to achieve cure in a high proportion of cases. The conditions we saw treated in this way were mainly 
neurological or musculoskeletal; and in general it seems that arthritis, neuritis, and paralysis after nerve injury or stroke are among the disorders treated most successfully. In these conditions the needles are stimulated electrically, usually with a square-wave alternating pulse at about 200 cycles per minute. The power source is a 6 volt battery. This stimulation causes obvious muscular twitching at the site of insertion of the needle but apparently no great discomfort for the patient.

Use of acupuncture for symptomatic relief is so much part of the way of life in China that most patients and many doctorsof both systems-expect it to be the treatment of first choice for the relief of pain or discomfort. We saw its use in the treatment of angina and were told that it is often used to relieve cardiac pain after myocardial infarction. Apparently it is used for its analgesic effect in childbirth, in migraine, and in many chronic skeletal disorders. There is obviously a distinction between this use of acupuncture and claims that it has a curative action: this is just one of the conflicts of opinion that has yet to be resolved.

\section{Acupuncture for Anaesthesia}

For many people inside and outside medicine the use of acupuncture for anaesthesia is the major point of interest in modern medicine in China. The British party included specialists in anaesthesia and neurophysiology, and we were therefore shown many operations done under acupuncture anaesthesia. These included operations on the thyroid, the eye, the heart, and the brain, and hernia repair. Several of the patients we saw walked into the operating theatre in our presence, and we were able to observe the insertion of needles and the questioning of the patient to determine that the required sensations were being felt. During operation the patients were in conversation with the acupuncturist, and several of the British party talked to patients (via an interpreter) during surgery. The surgeon's activity caused no apparent distress or discomfort to the patients. After the operation was completed the acupuncture needles were removed. We were told that the local anaesthetic effect at the operation site persisted for an hour or so, and the patients certainly appeared to remain free of pain. Several walked out of the operating theatre or waved to the British party as they left.

All the hospitals we visited use acupuncture for some operations, but for only a small fraction of the total. Some operations seem to be regarded as highly suitable for acupuncture; among these are thyroid and eye surgery and gynaecological procedures. Figures quoted varied from hospital to hospital, but in some at least more than half of the patients opt for acupuncture anaesthesia for this type of operation-and it is the patient who has to make a positive choice to have acupuncture.

Acupuncture is used far less for abdominal surgery and seems to be used very little for emergency operations or for the treatment of fractures. Some thoracic surgery is done under acupuncture: we saw operations on the mitral valve using this technique with the patient apparently unconcerned that his chest was open and one lung collapsed. Before operation the patient is taught breathing exercises and how to re-expand his lung after the chest has been closed, and it seems that these procedures are effective. However, in the last two years or so it seems that less than $10 \%$ of all chest surgery in specialist units has been done under acupuncture; and indeed taking the whole range of operations done in major hospitals there seems general agreement that about $10-15 \%$ are done under acupuncture. Epidural anaesthesia is also widely used: so are regional nerve blocks.

Chinese doctors are quite frank about the drawbacks of acupuncture anaesthesia. It is difficult to achieve adequate muscular relaxation for abdominal surgery, and manipulation of

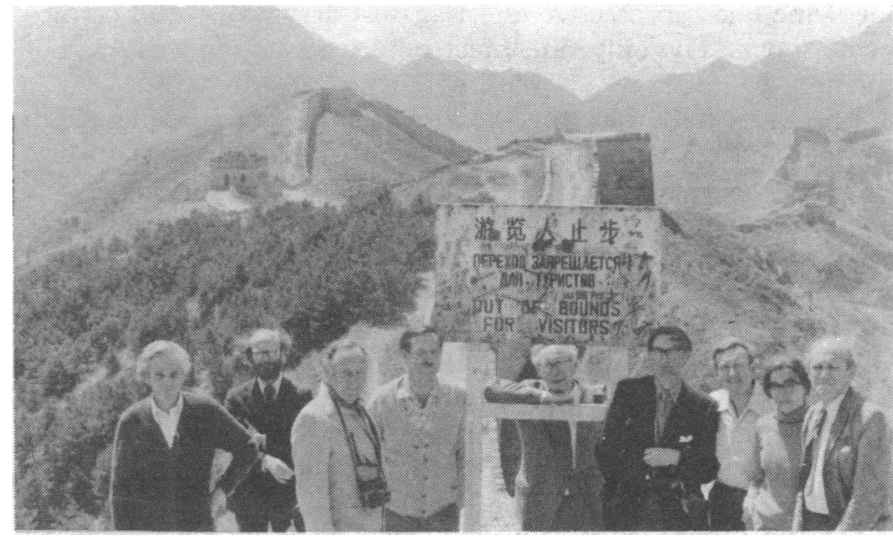

FIG. 4-Some of the members of the medical delegation on the Great Wall of China: Professor P. Alexander, Professor P. Wall, Mr. W. P. Cleland, Dr. P. O. Williams, Professor Sir David, Smithers, Professor J. G. Robson, Professor G. Hamilton Fairley, Dr. E. M. Adey, and Dr. J. F.
Nunn.

internal viscera by the surgeon can cause the patient to experience unpleasant sensations. In the operations we saw one or two of the patients seemed to experience some discomfort during the insertion of the needles. Against the drawbacks should be set the self-evident advantages of acupuncture anaesthesia: there is no risk of an anaesthetic death, and the technique prevents many postoperative complications such as chest infections.

One aspect of acupuncture anaesthesia that became apparent on the tour is its developing state. Thyroid operations, for example, seem to be done under acupuncture frequently, but some acupuncturists used needles in the arm while others used the ear, and it is clear that there is no uniform code of practice at present. The procedure has apparently been much simplified in recent years: at one time up to 30 needles might be used to achieve anaesthesia, but now it is commonplace for only two to be used. Electrical stimulation of the needles was used in all the cases we saw, but we were shown film of cases in which only manual rotation of the needles was employed.

\section{Conclusions}

Efforts are being made to refine the techniques used in acupuncture anaesthesia and to determine for which conditions it should be used. The success of the procedure has clearly given an enormous impetus to the reassessment by scientific methods of the range corpus of practices used in traditional medicine; and this process, which has only just begun, may be expected to provide medical scientists in China and the West with more surprises in the future.

The full delegation (fig. 4) was: Mr. W. P. Cleland, Professor J. F. Goodwin, Dr. E. Lawson McDonald (cardiologists); Professor G. Hamilton Fairley, Professor P. Alexander, Professor Sir David Smithers (onoologists); Professor P. Wall, Dr. J. F. Nunn, Dr. G. Hulands, Professor J. G. Robson (anaesthetists and neurophysiologists); Dr. P. O. Williams (Wellcome Trust); Dr. E. M. Adey, Dr. A. J. Smith (British Medical fournal).

\section{References}

1 Sidel, V. M., and Sidel, R., Serve The People, Observations on Medicine in the People's Republic of China. New York, Macy Foundation, 1973.

2 Risse, G. B., Modern China and Traditional Chinese Medicine, A SympoThomas, 1973. 